\title{
Two-photon laser-assisted device alteration in CMOS integrated circuits using linearly, circularly and radially polarized light
}

\author{
M. Rutkauskas, ${ }^{1},{ }^{*}$ C. Farrell, ${ }^{1}$ C. Dorrer,${ }^{2}$ K. L. Marshall, ${ }^{2}$ T. Crawford,${ }^{3}$ T. R. Lundquist,${ }^{3}$ \\ P. Vedagarbha, ${ }^{3}$ K. Erington, ${ }^{4}$ D. Bodoh ${ }^{4}$ and D. T. Reid ${ }^{1}$
}

${ }^{1}$ Scottish Universities Physics Alliance (SUPA), Institute of Photonics and Quantum Sciences, School of Engineering and Physical Sciences, Heriot-Watt University, Riccarton, Edinburgh EH14 4AS, UK

${ }^{2}$ Laboratory for Laser Energetics, University of Rochester, 250 E. River Road, Rochester, New York 14623-1299, USA

${ }^{3}$ DCG Systems Inc., 3400 West Warren Avenue, Fremont, California 94538, USA

${ }^{4}$ Freescale Semiconductor Inc., 6501 West William Cannon Drive, Austin, Texas 78735, USA

Corresponding author Mr. Marius Rutkauskas (mr309@hw.ac.uk)

\begin{abstract}
The rapidly developing semiconductor industry demands constant innovations in optoelectronic imaging of semiconductor integrated circuits to keep up with continuing device scaling. It was recently shown that two-photon laser-assisted device alteration (2pLADA) can deliver precision fault isolation. Here we describe an investigation into the influence of the incident light polarization on the 2pLADA spatial resolution. Linear polarization provides a highly confined but elliptical focal spot, while circular polarization diminishes the lateral resolution but benefits from a symmetrical focal spot. Radially polarized light potentially provides the highest lateral imaging resolution in all directions at the expense of the longitudinal resolution. By comparing 2pLADA results obtained using linear, circular and radial polarizations we show that certain polarizations have advantages in particular applications. Therefore a polarization optimized 2pLADA tool can achieve a sufficiently high performance to isolate faults of transistors separated by as little as $100 \mathrm{~nm}$ and maybe smaller.
\end{abstract}

\section{Keywords and OCIS codes}

(180.4315) Nonlinear microscopy; (260.5430) Polarization; (130.5990) Semiconductors.

\section{$[1 / 12]$}




\section{Introduction}

The progressive decrease in feature sizes of complementary metal-oxide semiconductor (CMOS) integrated circuits (ICs) means that diagnostic solutions for spatial fault localization within these devices must also be continuously refined to remain relevant for the prevailing technology node. Optical techniques - specifically the laser stimulation of semiconductor devices - encompass a wide variety of failure analysis methodologies such as optical beaminduced resistance change $(\mathrm{OBIRCH})$ [1], resistive inter-connect localization (RIL) [2], soft defect localization (SDL) [3], optical beam-induced current (OBIC) imaging [4] and laser-assisted device alteration (LADA) [5]. As feature sizes diminish, these techniques face an immediate and acute challenge to their ability to provide sufficient spatial resolution, since the wavelength of light inside silicon is typically $>300 \mathrm{~nm}$, more than an order of magnitude larger than the current production node [6].

We concentrate here on LADA, which is a well-established imaging/analysis technique used to isolate interaction sites of timing-critical transistors [7]. In its original form, LADA employed a continuous wave (CW) laser and therefore provided no detailed temporal information [8], however we recently showed how this limitation can be overcome by using two-photon absorption (TPA) induced by an infrared femtosecond laser [9]. In this earlier work we demonstrated how TPA, which generates electron-hole pairs within the active CMOS layer, could be synchronized with the internal clock of the device to allow a race condition to reveal speed-limiting transistor-switching evolution with a timing resolution below 10 ps [8].

In this paper we now address how the polarization state of the excitation light in two-photon LADA (2pLADA) influences the outcome of the technique. Previously, it was shown that the optical-beam induced current (OBIC) lateral resolution is significantly enhanced by TPA due to the effective narrowing of the point-spread function (PSF) associated with the nonlinear character of TPA [10]. The resolution achieved in sub-surface CMOS imaging is also considerably enhanced by using a solid-immersion lens (SIL) [11] and the highest resolutions can be achieved by combining TPA with SIL imaging [12]. Under the large values of numerical aperture (NA) associated with SIL microscopy it is known that the polarization state of the incident light plays a significant role in determining the image resolution [13]. The conventionally employed linearly polarized light forms an approximately elliptical focal spot [14], extended along the E-field vector of the light, implying that radially-polarized (RP) illumination could be used to further reduce the PSF area, since RP light experiences no equivalent PSF distortion under high-NA focusing [15]. 
In the following sections we detail the outcomes of 2pLADA imaging using various polarization states. We employed linear, circular and radial polarizations to investigate how 2pLADA imaging resolution depends on the E-field distribution of the incident light. The imaging system employed a liquid-crystal radial polarization converter (RPC) [16, 17] to generate a radially polarized beam with a high degree of mode purity, and in a way which allowed easy insertion and removal of the RPC to facilitate direct comparisons between different polarization states of the incident light.

\section{Femtosecond laser excitation source}

The 2pLADA was stimulated by 1280-nm pulses delivered from a source that used the Raman self-frequency shifting technique in a photonic crystal fiber (PCF) when pumped by a modelocked ytterbium-doped fiber laser (YDFL), which is shown in Fig. 1 [18]. The YDFL produced 1.3-ps chirped pulses at a nominal repetition frequency of $100 \mathrm{MHz}$, which could be modified over a range of $\sim 500 \mathrm{~Hz}$ by actuating a piezo-electric transducer, on which was mounted one of the intracavity fiber collimators. A fast electronic feedback loop was used to lock the repetition frequency of the laser to an external $100-\mathrm{MHz}$ clock supplied by the tester, while a slow loop which actuated an intracavity motorized-translation stage was used to maintain this lock over many hours in the presence of environmental changes. The laser pulses were compressed to durations of 160 fs by using a pair of Gires-Tournois interferometer mirrors, following which their average power was $350 \mathrm{~mW}$. Next, these de-chirped pulses were coupled with $75 \%$ efficiency into a highly nonlinear PCF. The resulting super-continuum contained chirp-free Raman-soliton pulses at the desired wavelength of $1280 \mathrm{~nm}$, which were then isolated by using a thin-film longpass filter with a cut-off wavelength of $1200 \mathrm{~nm}$. Finally, the filtered 1280-nm pulses were coupled into a polarization-maintaining delivery fiber, after which they were measured to have durations of $200 \mathrm{fs}$ and an average power of $20 \mathrm{~mW}$ (200 pJ). 


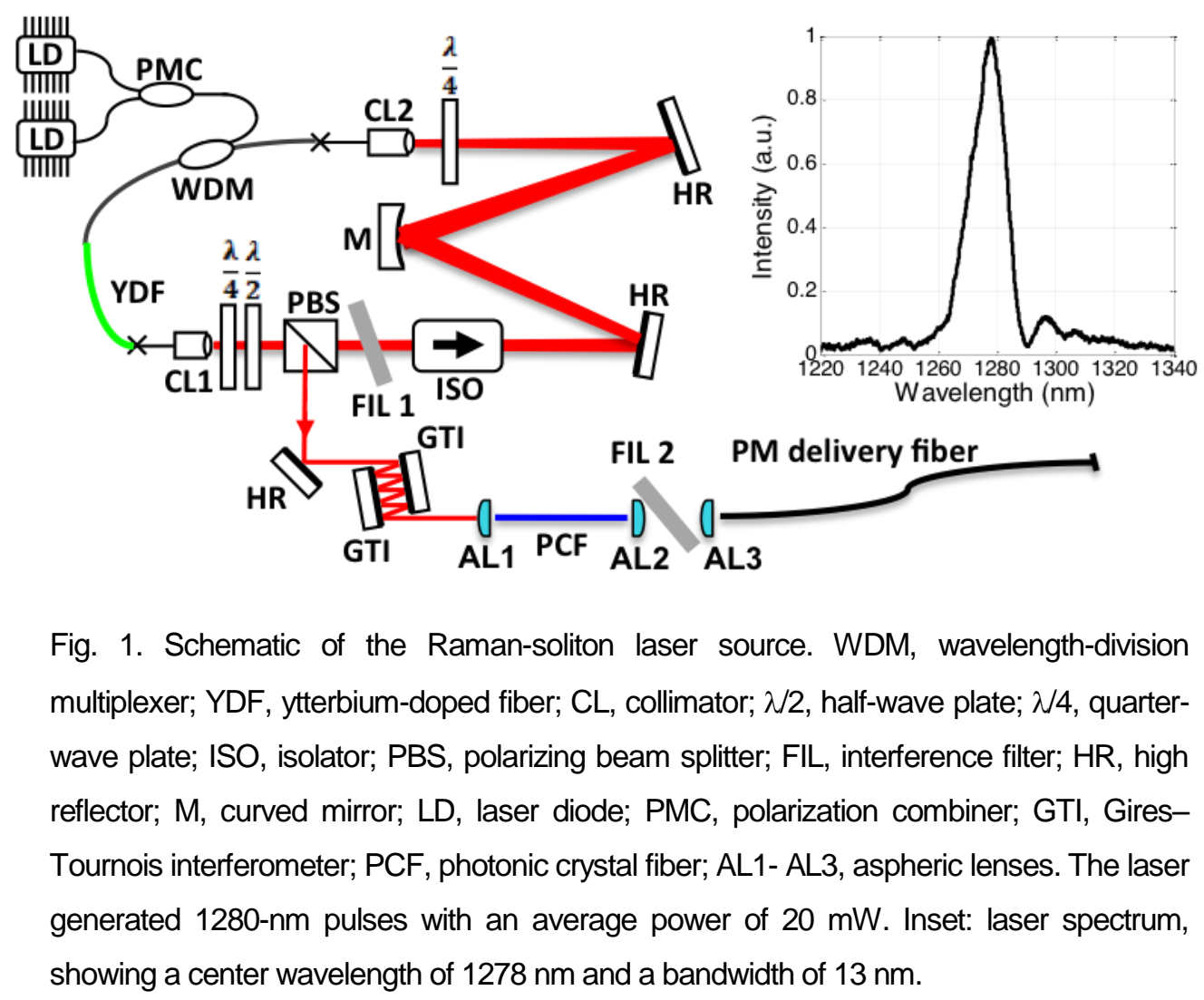

\section{Implementation of the 2pLADA microscope}

The experimental arrangement of the 2pLADA microscope is shown in Fig. 2. Femtosecond pulses of $10 \mathrm{pJ}$ energy and $195 \mathrm{GW} / \mathrm{cm}^{2}$ focal intensity at $1280 \mathrm{~nm}$ were delivered from the laser through a 4-m polarization maintaining fiber to a collimation module which presented a beam of diameter $4 \mathrm{~mm}$ into the telescope system used before the RPC. The collimation module also contained a beamsplitting cube used for the deflection of the returning laser beam to the detector. Lenses L1 and L2 formed a telescope used to increase the diameter of the beam before the RPC device, whose $20-\mathrm{mm}$ aperture made it necessary to use an expanded beam. A second telescope formed by lenses L3 and L4 then reduced the beam to a diameter matching the aperture of the galvanometer mirrors of the laser-scanning microscope (LSM) module. The LSM employed an integrated silicon SIL objective which provided 350× magnification and $N A=2.45$. A scalar calculation of the PSF full-width-half-maximum diameter using the Sparrow criterion for the 2pLADA microscope with $1280 \mathrm{~nm}$ incident wavelength and NA $=2.45$ provides a resolution of $260 \mathrm{~nm}$, however this value takes no account of the resolution improvement due to the effect of nonlinear absorption. 
The device under test (DUT) was a proprietary 28-nm-feature-size bulk silicon test device $\left(\mathrm{V}_{\mathrm{DD}}=0.8 \mathrm{~V}\right.$, clock frequency $\left.=50 \mathrm{MHz}\right)$. The device was engineered to perform a logic operation, which was previously described in [8]. By controlling the supply voltage the fail rate was set to $50 \%$ and typically several hundred 2pLADA images of a region of PMOS and NMOS transistors were recorded using a pixel dwell time of $32 \mu$ s and an image size of $512 \times 512$.

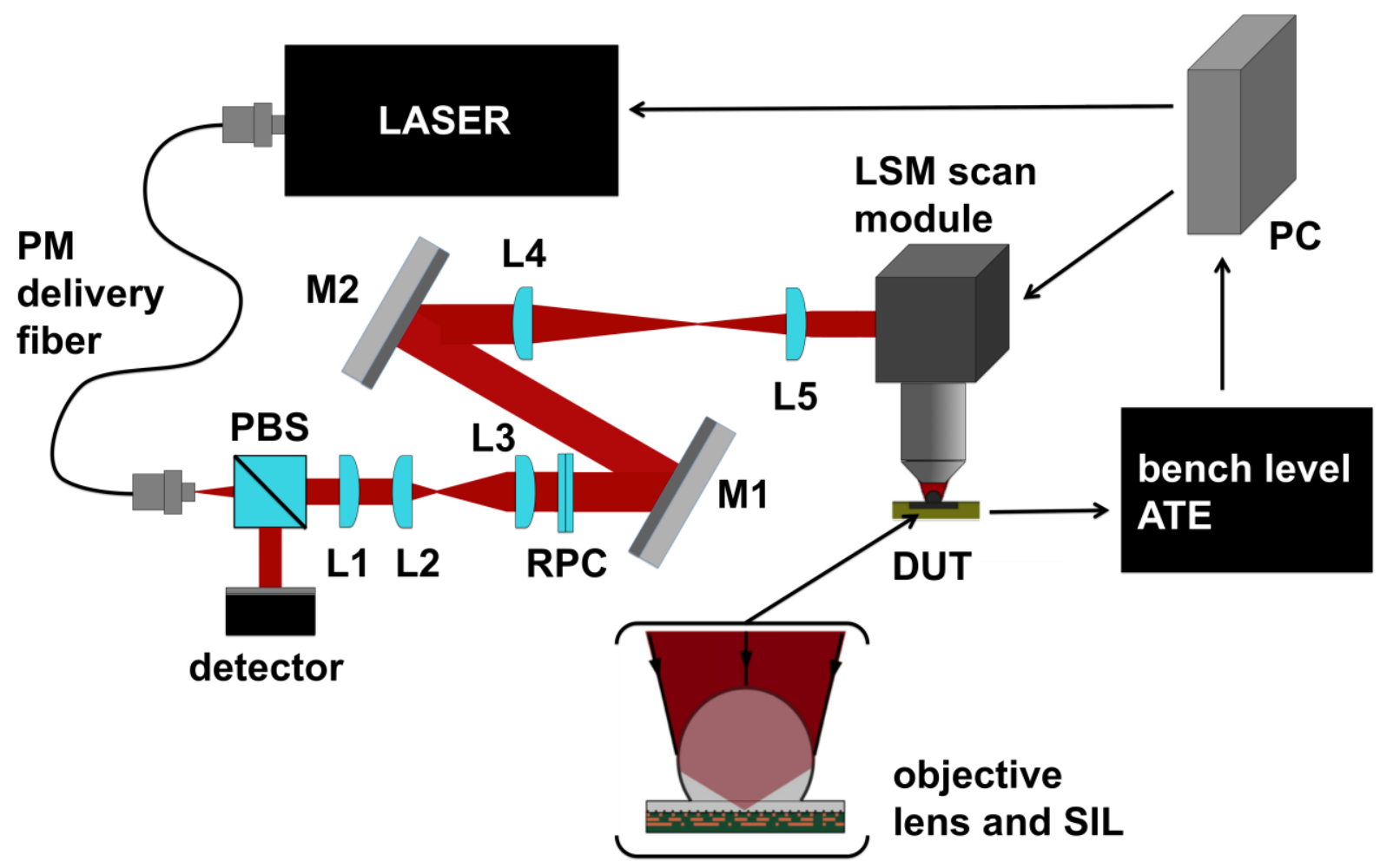

Fig. 2. $2 p L A D A$ microscope layout. The beam to the module is delivered using a polarization maintaining fiber, where it enters through a polarizing beam splitter (PBS). The PBS and its associated detector are used for confocal imaging to navigate to the area of interest in the DUT. After this, the beam is collimated using lens L1 and is expanded using lenses L2 and L3 and guided through the RPC device. Mirrors M1 and M2 are used to fold the layout and to deflect the beam into the reducing telescope (L4$L 5)$, which images it onto the galvanometer mirrors of the LSM scan module. The beam is then guided to the 350x silicon SIL microscope objective $(N A=2.45)$. A half-wave plate (HWP) and a quarter-wave plate (QWP) were used just before the microscope objective to prepare the incident polarization for the imaging experiments. 


\section{Results from 2pLADA imaging under different polarization conditions}

\subsection{Comparison of lateral localization resolutions}

2pLADA images were obtained using the microscope described in Section 3 by averaging several hundred frames, with an approximate acquisition time of 300 seconds. We also observed single-event upset (SEU) features, typically at intensity levels below those needed for 2pLADA. More information about SEUs observed during 2pLADA imaging is provided in [8]. Separate images were recorded using parallel linear, orthogonal linear, circular and radial polarizations. In Fig. 3 we present an overview image of a 2pLADA measurement for PMOS and NMOS transistors, showing the region of interest (red box) used for the quantitative measurements, which are described later.

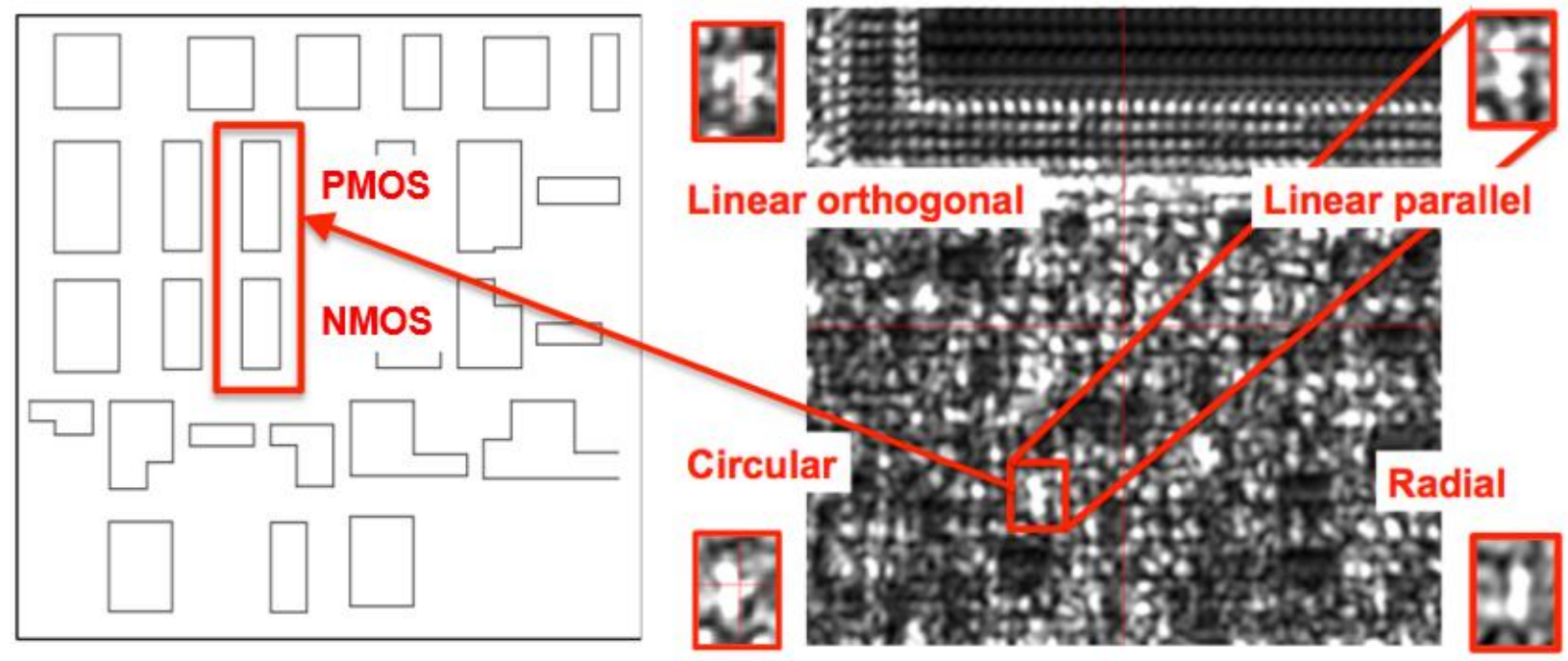

Fig. 3. Confocal image of the active area of the device. The zoomed-in area is shown for each of the different polarization states.

Figure 4 shows the 2pLADA images obtained under different incident polarization conditions for the region of interest indicated in Fig. 3. The images in the top row of Fig. 4 include the PMOS and NMOS transistors overlay from the CAD image as red rectangles, while the bottom row shows corresponding horizontal line-cuts, which allow localization resolution values to be inferred using a least-squares Gauss error function fit [11]. The CAD overlay indicates a separation between the transistors of $117 \mathrm{~nm}$, which appears to be most clearly resolved when linear polarization is used with its E-field parallel to the transistor channel. This is an expected result because the point spread function is longer in the direction parallel to the E-field and narrower in the orthogonal direction [13]. Comparable localization resolution also appears to be 
possible (Fig. 4, bottom row, column 4) when using radially polarized light, which in principle can provide the same PSF width as optimized linear polarization.
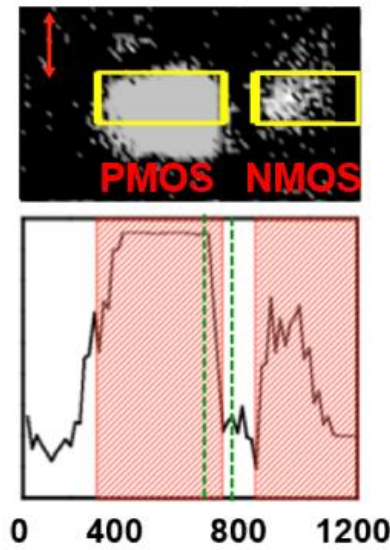

Position (nm)
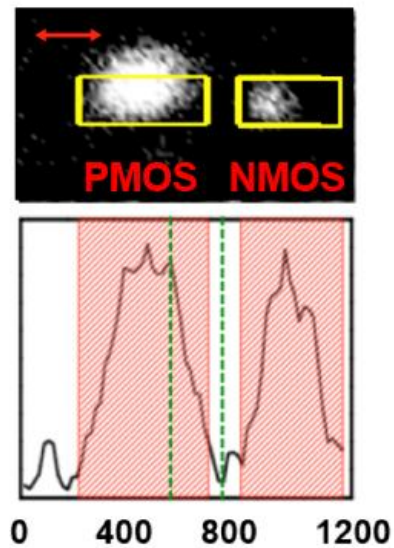

Position (nm)
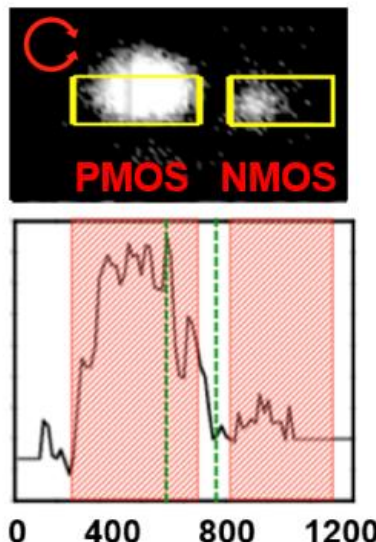

Position (nm)
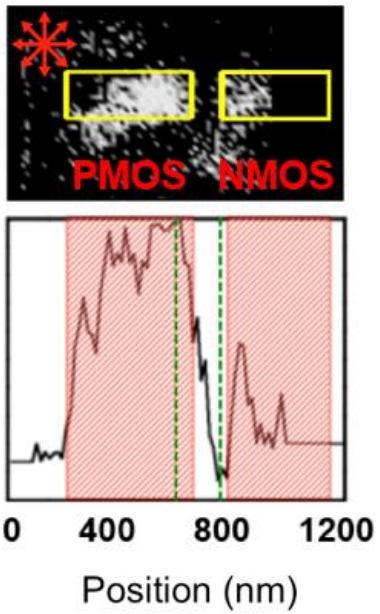

Fig. 4. Top row: $2 p L A D A$ images of PMOS and NMOS transistors obtained using 600 averages and the linear / circular / radial polarization states indicated in red. The rectangles show the PMOS and NMOS transistors overlay from the CAD image. Bottom row: corresponding absolute line-cut profiles of the active sites. The red shading depicts the transistor footprints from CAD and the green dashed lines show the region used for the localization resolution calculation (see text). The overlap integrals between the CAD layout and the 2pLADA images were normalized to the highest overlap value and were (left to right) $0.82,0.91,0.86$ and 1.0 .

We compared how closely the 2pLADA signals correlated with the transistor regions defined by the CAD layout by evaluating a normalized overlap integral between the 2pLADA signal and the area bounded by the left rectangle in the Fig. 4 images. The area in the right rectangle was not included in the calculation because in this region the 2pLADA signal was noisier and very sensitive to the relative delay of the laser pulses. We note also that the right rectangle corresponded to the NMOS well, which typically exhibited a weaker and more timing-critical LADA response [9]. The overlap integral was highest for the case of radial polarization and was normalized to 1.0, giving values for the other cases of $0.82,0.91$ and 0.86 (see Fig. 4). Despite the improved correlation between the CAD and the 2pLADA image for radial polarization, additional artifacts are evident when using RP illumination, for example there is substantial distortion and elongation of the image when compared with linear polarization case. It is well known that RP illumination exhibits a strong longitudinal component, which can be advantageous by making the focal positioning less critical. The same effect may cause the LADA signal to be generated over an extended depth in the DUT, resulting in a skew in the 
shape of the 2pLADA image, however the exact cause of the observed distortion is currently unclear. As expected, circular polarization resolves the gap between the transistors less well than with optimized linear polarization but with a performance similar to non-optimized linear polarization [14].

The transition of the 2pLADA signal across the gap between PMOS and NMOS transistors (indicated by the green dashed lines in Fig. 4) was used as the basis for making a quantitative estimate of the localization resolution in each case by fitting a Gaussian error function, as shown in Fig. 5. The line-cuts in the 2pLADA signal (Fig. 5) reveal that the gap between the transistors is best resolved for E-field polarizations parallel to the gap, as expected from theory [14] and previous two-photon OBIC experiments [13]. This polarization leads to a resolution of $99 \mathrm{~nm}$, which is increased by $46 \%$ to $144 \mathrm{~nm}$ when using the orthogonal linear polarization. Imaging using radially polarized light produced a resolution value of $120 \mathrm{~nm}$, the next lowest resolution after that obtained using optimized linear polarization. We note that this poorer RP resolution may be due to the purity of the RP state being less than $100 \%$. The measured value was $94 \%$ [19] and Yurt et al [20] have observed this can be a source of artifacts when using RP illumination. When circular polarization was used the resolution was degraded to $133 \mathrm{~nm}$.
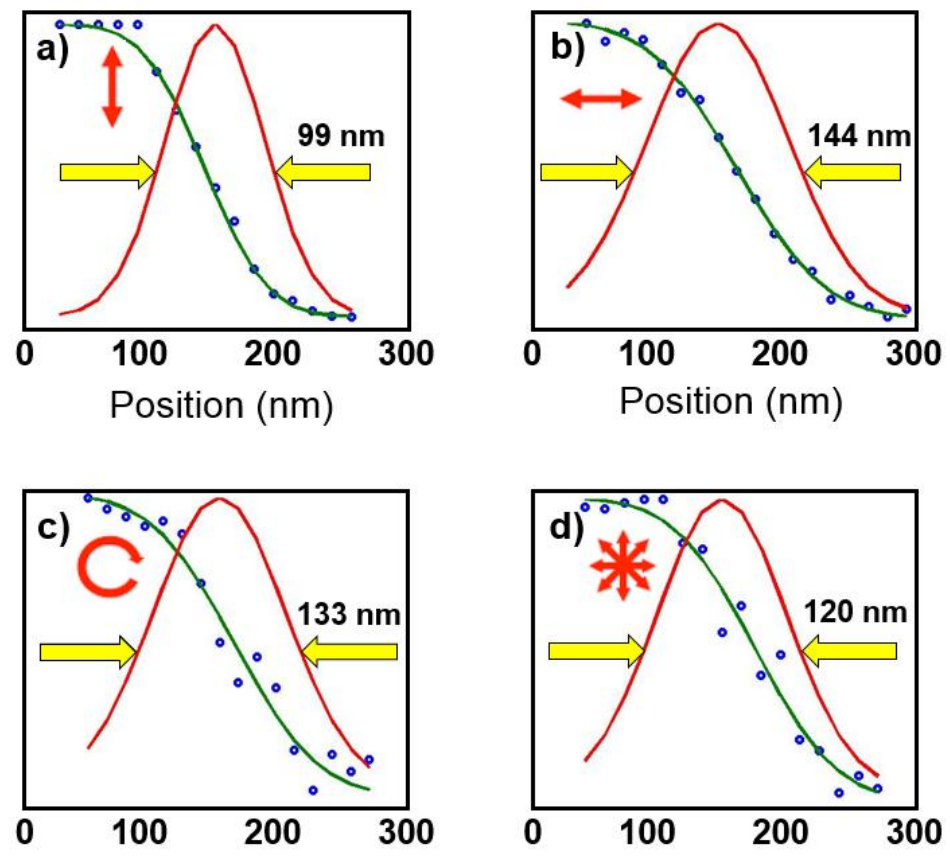

Position (nm)

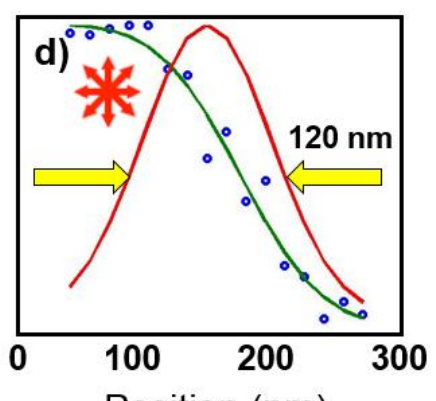

Fig. 5. Line-cuts of the 2pLADA images of the active area for different polarization states, recorded between the green dashed lines depicted in Fig. 4 and fitted with a Gauss error function fit. 
In summary, while the best localization resolution was obtained using linearly polarized and RP illumination, circular polarization benefits from a symmetrical focal spot and is free of the artifacts which are apparent when imaging using radial polarization.

\subsection{Sensitivity of 2pLADA to focusing and timing}

The carrier injection responsible for 2pLADA occurs due to two-photon absorption, an effect whose strength depends on the intensity of the laser pulses. Consequently, a strong sensitivity to focal position is expected because of the associated change in the area of the focused beam in the device.

The sensitivity to the displacement from focal position was investigated by changing the relative position between the backing objective and the integrated SIL. The focal position itself was not found to be strongly intensity dependent. As Fig. 6 illustrates, the 2pLADA localization resolution displayed a clear minimum over a region of around $3 \mu \mathrm{m}$, corresponding to a change in the focal position inside the device of around $250 \mathrm{~nm}$. These data provided a means of ensuring that the focal position had been repositioned consistently in the DUT as these localization resolution measurements were repeated using different polarization states.

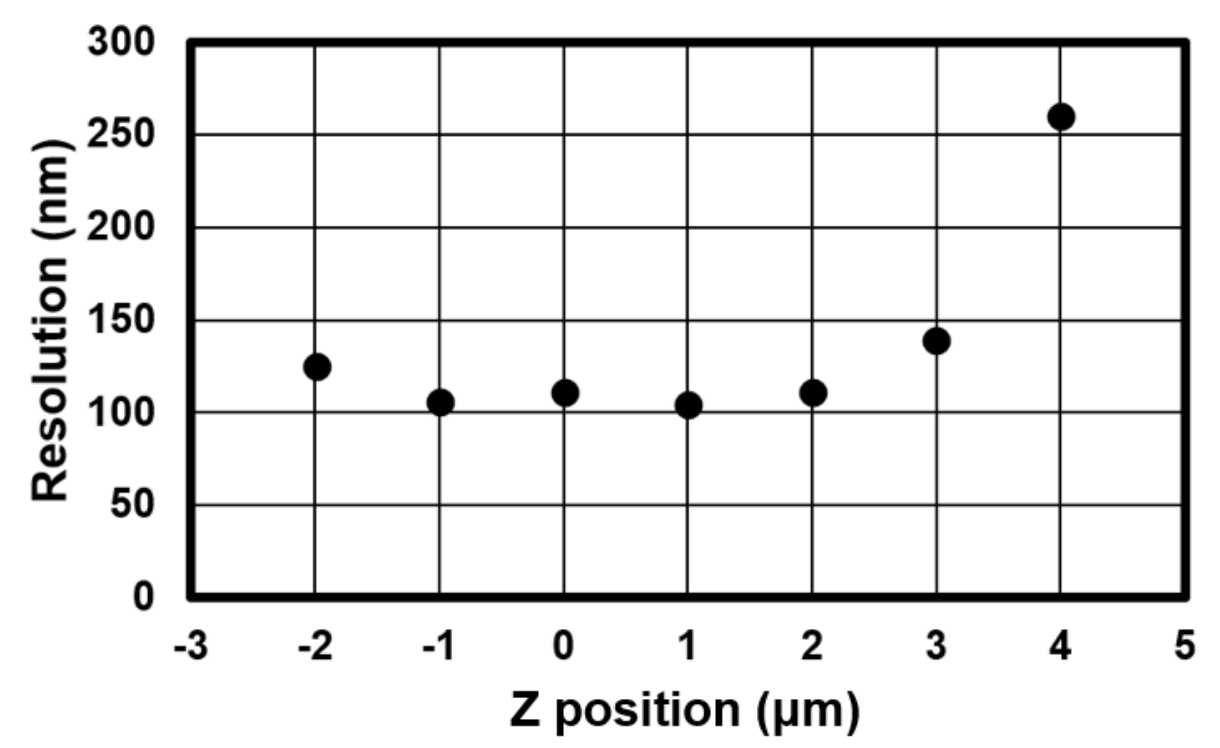

Fig. 6. Example of the 2pLADA lateral resolution dependence on the $z$ position of the DUT, obtained using parallel linear polarization.

The other novel aspect of 2pLADA is its sensitivity to the relative timing between the laser pulses and the clock signal within the DUT, an important feature which uniquely provides detailed temporal information about the switching of individual transistors. It was therefore essential to synchronize the laser pulse arrival with the transistor switching event occurring at 
the 2pLADA site. The electrical-system jitter was around $10 \mathrm{ps}$ [8] and the modelocked laser was phase-locked to the DUT clock with a much lower pulse-to-pulse jitter, typically of order one femtosecond. The synchronization system provided an adjustable phase between the electrical and optical pulses with a nominal resolution of $3 \mathrm{ps}$, sufficient to ensure the optical and electrical pulses were coincident in time. We investigated this by recording a number of 2pLADA images as the delay was adjusted, then extracting corresponding localization resolution values from these images. As can be seen from Fig. 7, delay adjustments of $<10$ ps led to a noticeable degradation of the localization resolution from its minimum value.

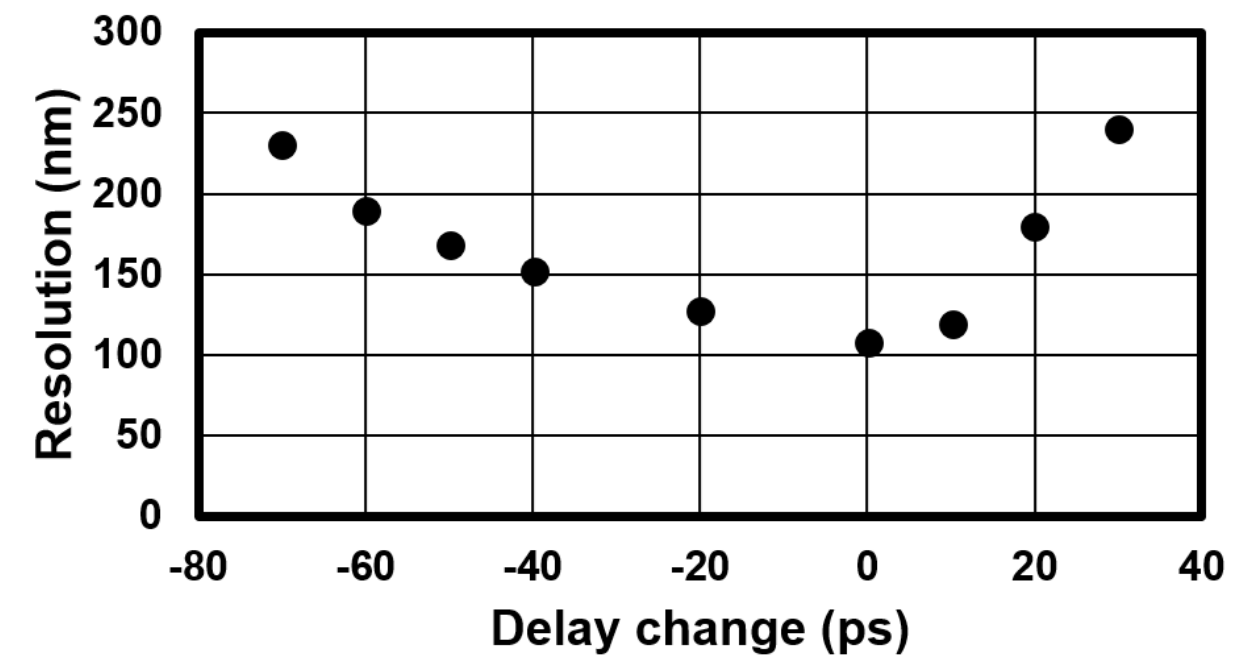

Fig. 7. Example of the delay dependence of the 2pLADA lateral resolution, obtained using parallel linear polarization.

\section{Conclusions}

The results presented here represent the first detailed assessment of the role of laser polarization in 2pLADA imaging. Linear polarization showed a clear advantage for analyzing advanced ICs as long as the preferred polarization direction could be identified, which in fact can easily be established if the polarization is readily rotatable, e.g. using a half-wave plate. The linear and radial polarizations provided the best lateral localization resolutions compared to the circular polarization. In principle, radially polarized light should have the advantage of producing a PSF with circular symmetry, in contrast to the approximately elliptical shape generated in linear polarization case. However, our results using radial polarization indicated a distortion of the 2pLADA signature, which appears to be a measurement artifact which is not observed in $2 p L A D A$ images recorded with the other polarization states. This effect may result from the 
strong longitudinal E-field component of the radially polarized beam interacting with deeper features in the DUT, however more measurements are needed to understand the origin of this behavior. In less advanced process technologies circular polarization may be useful due to its symmetric performance and absence of artifacts, even though it provides poorer localization resolution. Nevertheless radial polarization still promises even better resolution as it is more amenable than other polarizations when incorporating apodization which would then outperform linear polarization under conditions of NA higher than the value of 2.45 used in this work.

\section{Acknowledgements}

This work was supported by the Intelligence Advanced Research Projects Activity (IARPA) via Air Force Research Laboratory (AFRL) Contract No. FA8650-11-C-7104. The U.S. Government is authorized to reproduce and distribute reprints for Governmental purposes notwithstanding any copyright annotation thereon. The views and conclusions contained herein are those of the authors and should not be interpreted as necessarily representing the official policies or endorsements, either expressed or implied, of IARPA, AFRL, or the U.S. Government. This material is based upon work partially supported by the Department of Energy National Nuclear Security Administration under Award Number DE-NA0001944, the University of Rochester, and the New York State Energy Research and Development Authority. The support of DOE does not constitute an endorsement by DOE of the views expressed in this article.

\section{References}

1. K. Nikawa and S. Inoue, IEEE International Reliability Physics Proceedings - 34th Annual, 346- 354 (1996).

2. E. I. Cole, P. Tangyunyong, C. F. Hawkins, M. R. Bruce, V. J. Bruce, R. M. Ring, and W. L. Chong, in ASM International Symposium for Testing and Failure Analysis - 27th Annual, 43-50 (2001).

3. M. R. Bruce, V. J. Bruce, D. H. Eppes, J. Wilcox, E. I. Cole, Jr., P. Tangyunyong, and C. F. Hawkins, "Soft defect localization (SDL) in ICs" ASM International Symposium for Testing and Failure Analysis - 28th Annual, ASM International, 21-27 (2002).

4. E. I. Cole, J. M. Soden, J. L. Rife, D. L. Barton, and C. L. Henderson, IEEE International Reliability Physics Proceedings - 32nd Annual, 388-398 (1994).

5. J. Rowlette and T. Eiles, ITC International Test Conference 1, 264-273 (2003).

6. A. Douin, V. Pouget, M. De Matos, D. Lewis, P. Perdu, and P. Fouillat, Microelectron. Reliab. 46, 1514-1519 (2006). 
7. K. A. Serrels, C. Farrell, T. R. Lundquist, D. T. Reid, and P. Vedagarbha, Appl. Phys. Lett. 99, 193103 (2011).

8. K. A Serrels, K. Erington, D. Bodoh, C. Farrell, N. Leslie, T. R. Lundquist, P. Vedagarbha, and

D. T. Reid, Opt. Express 21, 29083-29089 (2013).

9. K. Erington, D. Bodoh, K. A. Serrels, C. Nemirow, N. Leslie, T. R. Lundquist, P. Vedagarbha, C. Farrell and D. T. Reid, Reliability Physics Symposium, 2014 IEEE International, 3F.4.1 3F.4.5 (2014).

10. C. Xu and W. Denk, Appl. Phys. Lett. 71, 2578-2580 (1997).

11. S. B. Ippolito, B. B. Goldberg, M. S. Unlu, Appl. Phys. Lett. 78, 4071 (2001).

12. K. A. Serrels, E. Ramsay, P. A. Dalgarno, B. D. Gerardot, J. A. O'Connor, R. H. Hadfield, R. J. Warburton and D. T. Reid, J. Nanophoton. 2, 021854 (2008).

13. K. A. Serrels, E. Ramsay, R. J. Warburton and D. T. Reid, Nature Photon. 2, 311 - 314 (2008).

14. Q. Zhan, Advances in Optics and Photonics 1, 1-57 (2009).

15. R. Dorn, S. Quabis, and G. Leuchs, Phys. Rev. Lett. 91, 233901 (2003).

16. M. Stalder and M. Schadt, Opt. Lett. 21, 1948-1950 (1996).

17. M. Statt, M. Vargas, J. Oliver, S. Chen, K. Marshall, and C. Dorrer, Conference on Lasers and Electro-Optics 2012, OSA Technical Digest (online) (Optical Society of America, 2012), paper JTh2A.56.

18. C. Farrell, K. A. Serrels, T. R. Lundquist, P. Vedagarbha, and D. T. Reid, Opt. Lett. 37, 17781780 (2012).

19. M. Rutkauskas, C. Farrell, C. Dorrer, K. L. Marshall, T. R. Lundquist, P. Vedagarbha, and D. T. Reid, Conference on Lasers and Electro-Optics 2015, OSA Technical Digest (online) (Optical Society of America, 2015), STh1H.7.

20. A. Yurt, M. D. W. Grogan, S. Ramachandran, B. B. Goldberg and M. S. Ünlü, Optics Express 22, 7320-7329 (2014). 

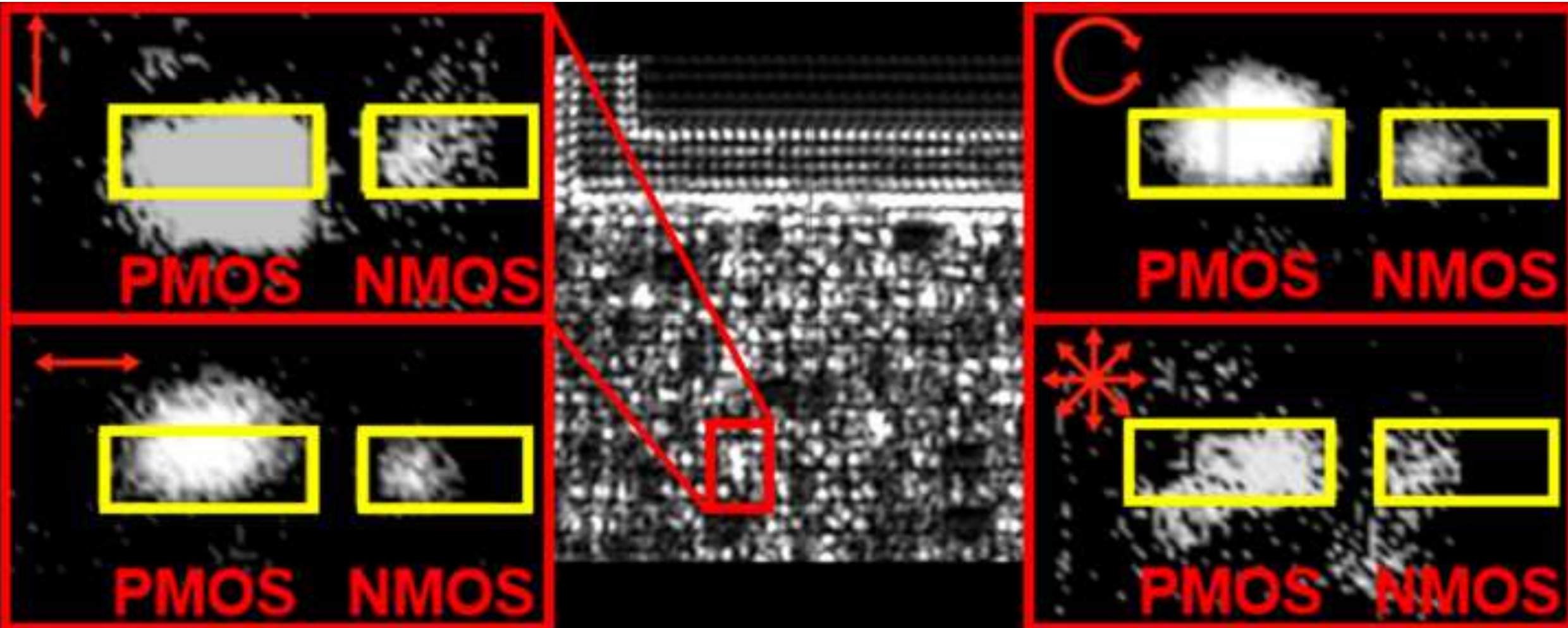

\section{PMÓS NMOS}

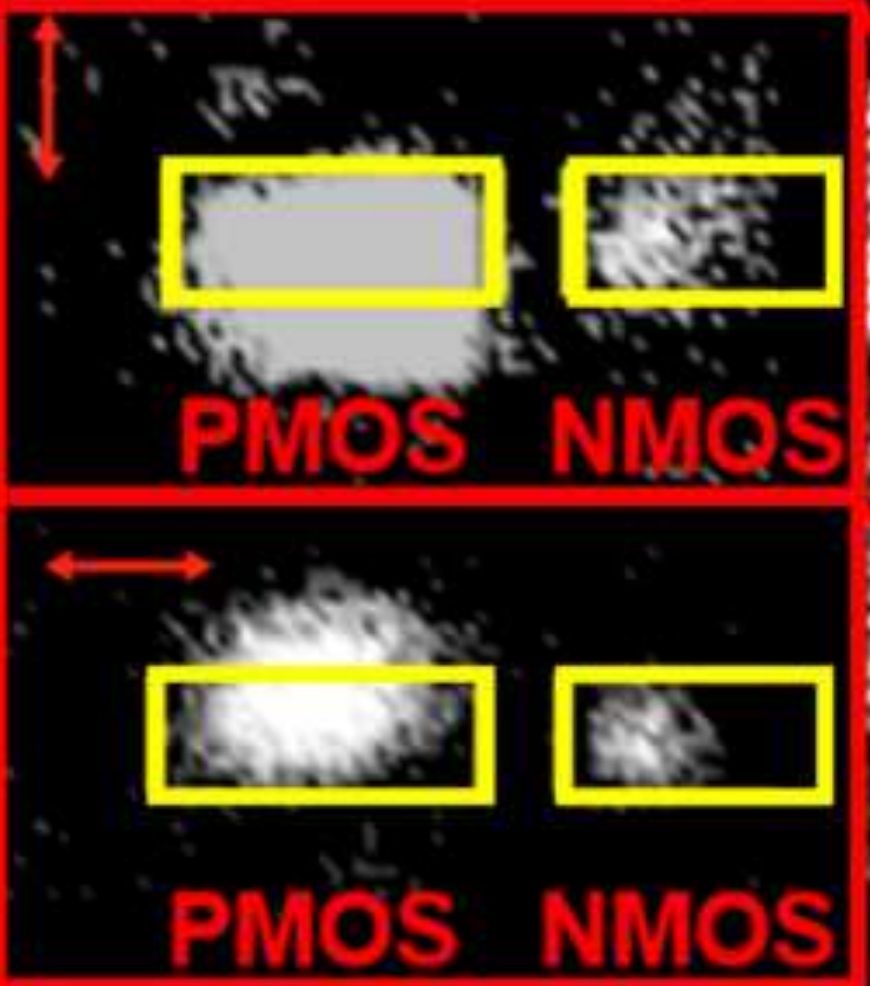

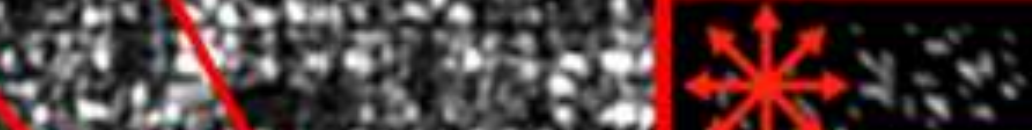

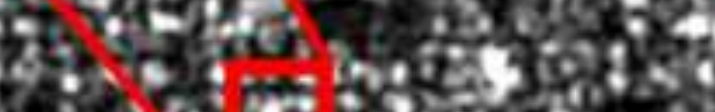

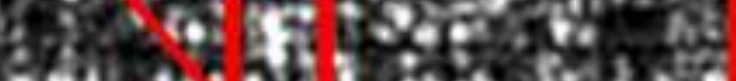

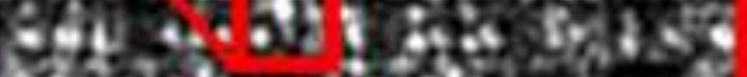

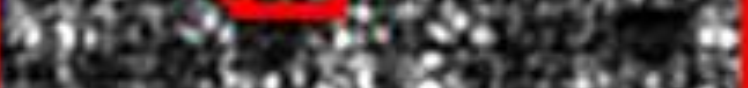

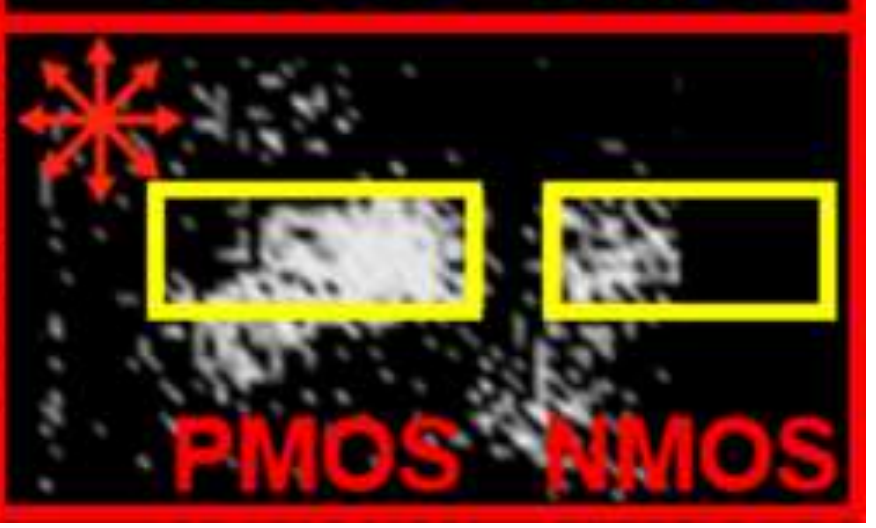

